\title{
Risk stratification in unstable angina and non-Q wave myocardial infarction using soluble cell adhesion molecules
}

\author{
N T Mulvihill, J B Foley, R T Murphy, R Curtin, P A Crean, M Walsh
}

\begin{abstract}
Objective-To assess prospectively the prognostic value of soluble cellular adhesion molecules (CAMs) in patients with unstable angina and non- $\mathrm{Q}$ wave myocardial infarction and to compare their prognostic accuracy with that of $\mathrm{C}$ reactive protein (CRP).

Design and setting-Prospective observational study of patients presenting acutely with unstable angina and non-Q wave myocardial infarction to a single south Dublin hospital.

Methods-Patients with Braunwald IIIA unstable angina and non-Q wave myocardial infarction had serum samples taken at presentation before initiation of antithrombotic treatment and were followed for six months. The primary end point was the occurrence of major adverse cardiovascular events (recurrent unstable angina, non-fatal myocardial infarction, and cardiovascular death) at six months. Concentrations of soluble intercellular adhesion molecule-1 (sICAM-1), soluble vascular cell adhesion molecule-1 (sVCAM-1), soluble endothelial selectin, and soluble platelet selectin were measured using an enzyme linked immunosorbent assay technique. CRP was measured with an immunophelometric assay.

Results-91 patients (73 men and 18 women, mean (SD) age 61 (11) years) were studied; 27 patients $(30 \%)$ had major adverse cardiac events during the six months of follow up. Concentration of CRP were significantly raised in patients who had an ischaemic event (mean (SEM) 11.5 (6.4) $\mathrm{mg} / \mathrm{l} v 5.4(2.5) \mathrm{mg} / \mathrm{l}, \mathrm{p}<0.001)$. Concentrations of sVCAM-1 were also significantly raised in the ischaemic event group (979 (30) $\mathrm{ng} / \mathrm{ml} v 729$ (22) $\mathrm{ng} / \mathrm{ml}, \mathrm{p}<0.001$ ). Both sVCAM-1 and CRP concentrations correlated strongly with the occurrence of an adverse event. The sensitivity of CRP $>3 \mathrm{mg} / 1$ and sVCAM-1 $>780 \mathrm{ng} / \mathrm{ml}$ for predicting future events was $>90 \%$. There was no difference in concentrations of sICAM-1, soluble endothelin selectin, or soluble platelet selectin between event and non-event groups.

Conclusion-Raised concentrations of sVCAM-1 and CRP are predictive of an increased risk of major adverse cardiovascular events six months after presentation with unstable angina and non-Q wave myocardial infarction. These findings suggest that the intensity of the vascular inflammatory process at the time of presentation is a determinant of clinical outcome in unstable coronary artery disease.

(Heart 2001;85:623-627)
\end{abstract}

Key words: cell adhesion molecules; risk stratification; unstable angina

Coronary plaque rupture, with subsequent platelet aggregation and thrombosis, is the key mechanism by which atherosclerosis leads to the acute coronary syndromes of unstable angina (UA), acute myocardial infarction (MI), and sudden cardiac death. ${ }^{1-3}$ There is a growing body of evidence implicating inflammation as an important triggering event in the pathogenesis of acute coronary syndromes. ${ }^{4}$ Local inflammatory cells generate and release cytokines, which activate endothelium, transforming its natural antiadhesive and anticoagulant properties. Evidence of an inflammatory process in acute coronary syndromes comes from a variety of sources including histological analysis of atherosclerotic coronary plaques, ${ }^{5}$ identification of activation of circulating leucocytes, ${ }^{67}$ and the observation of systemic release of thromboxanes and leukotrienes. ${ }^{8}$ Raised concentrations of $\mathrm{C}$ reactive protein (CRP) and serum amyloid $\mathrm{A}$ have been reported in patients with UA and in acute $\mathrm{MI},{ }^{9}{ }^{10}$ and increased concentrations of CRP at the time of hospital admission are predictive of an adverse outcome in patients with UA. ${ }^{11}$
The inflammatory cytokines generated induce increased expression of cellular adhesion molecules (CAMs). ${ }^{12}$ These CAMs have a crucial role in the transendothelial migration of leucocytes from the blood to the arterial intima. Circulating forms are detectable in serum and are referred to as soluble CAMs. ${ }^{13}$ Concentrations of three soluble CAMssoluble intercellular adhesion molecule-1 (sICAM-1), soluble vascular cell adhesion molecule-1 (sVCAM-1), and soluble endothelial selectin (sE-selectin) - are raised in patients with UA and non-Q wave MI (NQMI) up to six months after the initial ischaemic event. ${ }^{14}$ The aim of this study was to assess and compare prospectively the prognostic value of $\mathrm{CRP}$ and soluble CAMs in patients presenting with UA and NQMI.

\section{Methods}

PATIENTS

The study protocol was approved by the institutional ethics review committee and written informed consent was obtained from all participating patients. We enrolled patients 
Table 1 Clinical characteristics of the study patients

\begin{tabular}{llll}
\hline & No event $(n=64)$ & Event $(n=27)$ & $p$ Value \\
\hline Age (years) & $60(10)$ & $65(11)$ & $<0.02$ \\
Sex (M/F) & $51 / 13$ & $22 / 5$ & NS \\
Previous myocardial infarction (\%) & $26(41)$ & $11(41)$ & NS \\
Hypertension (\%) & $22(34)$ & $12(44)$ & NS \\
Diabetes (\%) & $10(16)$ & $5(19)$ & NS \\
Smoking (\%) & $29(45)$ & $13(48)$ & NS \\
Hypercholesterolaemia (\%) & $31(48)$ & $13(48)$ & NS \\
Revascularisation (\%) & $27(42)$ & $11(41)$ & NS \\
$\quad$ PTCA & $18(28)$ & $9(33)$ & \\
CABG & $9(14)$ & $2(7)$ & \\
\hline
\end{tabular}

PTCA, percutaneous transluminal coronary angioplasty; CABG, coronary artery bypass graft.

presenting acutely with UA and NQMI. UA was defined according to the Braunwald classification. ${ }^{15}$ UA patients had ischaemic chest pain at rest within the preceding 48 hours that had developed in the absence of an extracardiac precipitating cause with either ST segment depression of $\geqslant 0.1 \mathrm{mV}$ or $\mathrm{T}$ wave inversion in two or more contiguous leads on the presenting 12 lead ECG. Patients in the NQMI group had similar diagnostic criteria along with elevation of serum creatine kinase, without the evolution of pathological Q waves.

PROTOCOL

Patients were entered into the study between December 1997 and November 1998 at a single institution. Study patients had peripheral venous blood samples drawn at presentation. These samples were centrifuged and serum was stored at $-70^{\circ} \mathrm{C}$ for analysis at a later date. Decisions regarding clinical management were made by the attending cardiologist and revascularisation procedures were ischaemia driven either because of recurrent ischaemia on reasonable medical treatment or because of easily induced ischaemia during non-invasive testing.

SOLUBLE CELL ADHESION MOLECULE ASSAY Quantitative detection of serum concentrations of sICAM-1, sVCAM-1, sE-selectin, and soluble platelet selectin (sP-selectin) was performed using an enzyme linked immunosorbent assay (ELISA) technique employing a commercially available assay kit (R\&D Systems, Abingdon, UK). These assays use an immunoenzymometric technique for quantification of the soluble CAM present in the sample. This involves the simultaneous reaction of the soluble CAM with two antibodies directed against different epitopes on the specific soluble adhesion molecule. Measurements were repeated in $33 \%$ of the samples to determine the reproducibility of the measure. CRP concentrations were measured using a high sensitivity immunophelometric assay.

FOLLOW UP

Patients were reviewed three and six months after discharge. Major adverse cardiovascular events were classified as recurrent UA, nonfatal MI, and cardiovascular death. Recurrent UA was defined as either persistent ischaemic rest pain despite adequate medical treatment, or a new episode of UA with new ischaemic ECG changes necessitating readmission to
Table 2 Timing of major adverse cardiovascular events

\begin{tabular}{llll}
\hline & $\begin{array}{l}\text { Death } \\
(n=6)\end{array}$ & $\begin{array}{l}\text { Non-fatal MI } \\
(n=7)\end{array}$ & $\begin{array}{l}\text { RUA } \\
(n=14)\end{array}$ \\
\hline Days 0-30 & 4 & 6 & 3 \\
Days 31-60 & 1 & 0 & 3 \\
Days 61-90 & 1 & 1 & 3 \\
Days 91-120 & 0 & 0 & 2 \\
Days 121-150 & 0 & 0 & 2 \\
Days 151-180 & 0 & 0 & 1 \\
\hline
\end{tabular}

RUA, recurrent unstable angina

hospital. These clinical end points were mutually exclusive. Coronary revascularisation was reported as a secondary end point.

\section{STATISTICAL ANALYSIS}

The study population was divided into those who had a major adverse cardiovascular event in the six months following acute presentation and those who did not. Concentrations of soluble CAMs were measured in $\mathrm{ng} / \mathrm{ml}$ and expressed as mean (SEM). Single factor analysis of variance was used to compare concentrations of soluble CAMs and concentrations of CRP between event and non-event groups. Student's $t$ test was used to compare the incidence of common cardiovascular risk factors between the event and non-event groups; a $1 \%$ level of significance was used to correct for multiple comparisons. Multiple regression analysis was used to evaluate the correlation between concentrations of CRP, concentrations of soluble cell adhesion molecules, and multiple cardiovascular risk factors including age, sex, hypertension, diabetes mellitus, hypercholesterolaemia, and smoking with occurrence of an ischaemic end point. Concentrations of CRP and of soluble CAMs, and the risk factors outlined, were analysed as continuous variables with ischaemic events as the dependant variable. A probability value of $p \leqslant 0.05$ or less was considered significant. Receiver operator curves were used to identify risk stratifying cut off concentrations of soluble CAMs and CRP. Statistical analysis was performed using an SPSS software package (SPSS Inc, Chicago, USA).

\section{Results}

Ninety one patients were entered into the study (male to female ratio, 73:18, mean (SD) age 61 (11) years, 54 UA and 37 NQMI). During the six month follow up six patients died suddenly, seven suffered a non-fatal MI, and 14 had recurrent UA, for a major adverse cardiovascular event rate of $30 \%$. The study patients were divided into event $(n=27)$ and non-event groups $(n=64)$. The cardiovascular risk factor profiles were similar in the event and non-event groups (table 1). However, patients who had a major adverse cardiovascular event were older (65 (12) v 60 (11) years, p < 0.02). The majority of ischaemic events occurred within 30 days of the initial episode of UA or NQMI (table 2). The revascularisation rate during the study was $42 \%$ for patients without ischaemic events (18 percutaneous transluminal coronary angioplasty (PTCA) and nine coronary artery bypass graft (CABG) procedures) and $41 \%$ for those who had events (nine PTCA and two 
Table 3 Cardiovascular drug treatment

\begin{tabular}{llll}
\hline & No event $(n=64)$ & Event $(n=27)$ & $p$ Value \\
\hline Aspirin (\%) & $57(89)$ & $24(89)$ & NS \\
Intravenous heparin (\%) & $62(97)$ & $26(96)$ & NS \\
B Blockers (\%) & $40(63)$ & $13(48)$ & NS \\
Calcium channel blockers (\%) & $25(39)$ & $14(52)$ & NS \\
Nitrates (\%) & $28(44)$ & $13(48)$ & NS \\
ACE inhibitors (\%) & $17(27)$ & $11(41)$ & NS \\
HMG-CoA inhibitors (\%) & $26(41)$ & $13(48)$ & NS \\
\hline
\end{tabular}

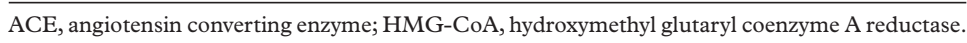

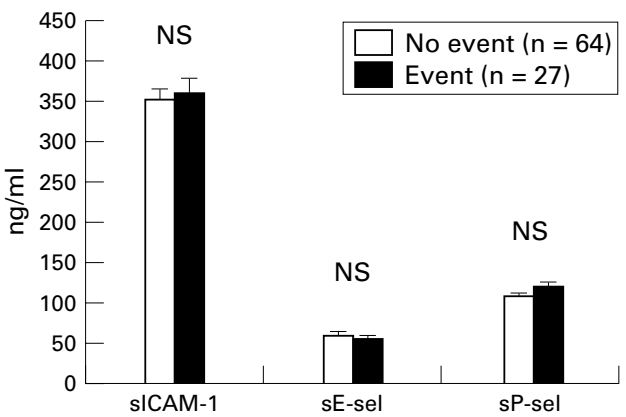

Figure 1 Concentrations of soluble intercellular adhesion molecule-1 (sICAM-1), soluble endothelial selectin

$(s E-s e l)$, and soluble platelet selectin $(s P$-sel) at presentation in event and non-event groups.

CABG). Fourteen PTCA and two CABG procedures were performed on patients in the non-event group during the period of initial hospitalisation. In the group of patients who had an ischaemic event, four had revascularisation procedures (three PTCA and one CABG) during the initial hospitalisation and a further seven required revascularisation (six PTCA and one $\mathrm{CABG}$ ) during follow up for recurrent UA. There were no deaths among patients undergoing revascularisation procedures. The antiplatelet, antianginal, and lipid lowering treatments were similar in both groups (table 3).

SOLUBLE CELL ADHESION MOLECULES

There was no significant difference in concentrations of sICAM-1, sE-selectin, or sP-selectin between event and non-event groups (350 (14) v 351 (20) ng/ml, NS for sICAM-1; 57 (3) v 59 (5) $\mathrm{ng} / \mathrm{ml}$, NS for sE-selectin, and 110 (9) v 119 (11) ng/ml, NS for sP-selectin) (fig 1). However, concentrations of sVCAM-1 at presentation were significantly raised in patients who went on to have ischaemic events during the six months of follow up (979 (30) $v 729$ (22) $\mathrm{ng} / \mathrm{ml}, \mathrm{p}<0.001$, respectively) (fig 2). Concentrations of sVCAM-1 remained significantly higher in the event group when we added revascularisation as a combined end point compared with patients who had neither a follow up event nor revascularisation (839 (26) $v 751$ (27) ng/ml, p < 0.05, respectively).

SOLUBLE VCAM-1

The patients were divided into two groups according to whether their sVCAM-1 concentrations were below or above $780 \mathrm{ng} / \mathrm{ml}$. This concentration was chosen because it had the highest sensitivity and specificity for predicting an adverse outcome. A total of 44 patients $(48 \%)$ had a concentration of sVCAM-1

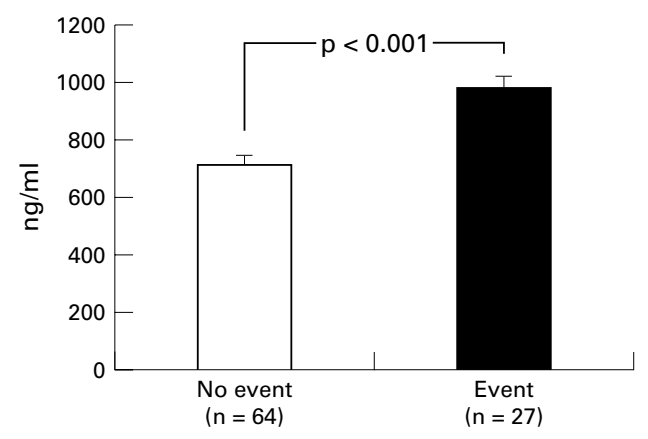

Figure 2 Concentrations of soluble vascular cell adhesion molecule-1 (sVCAM-1) at presentation in event and non-event groups.
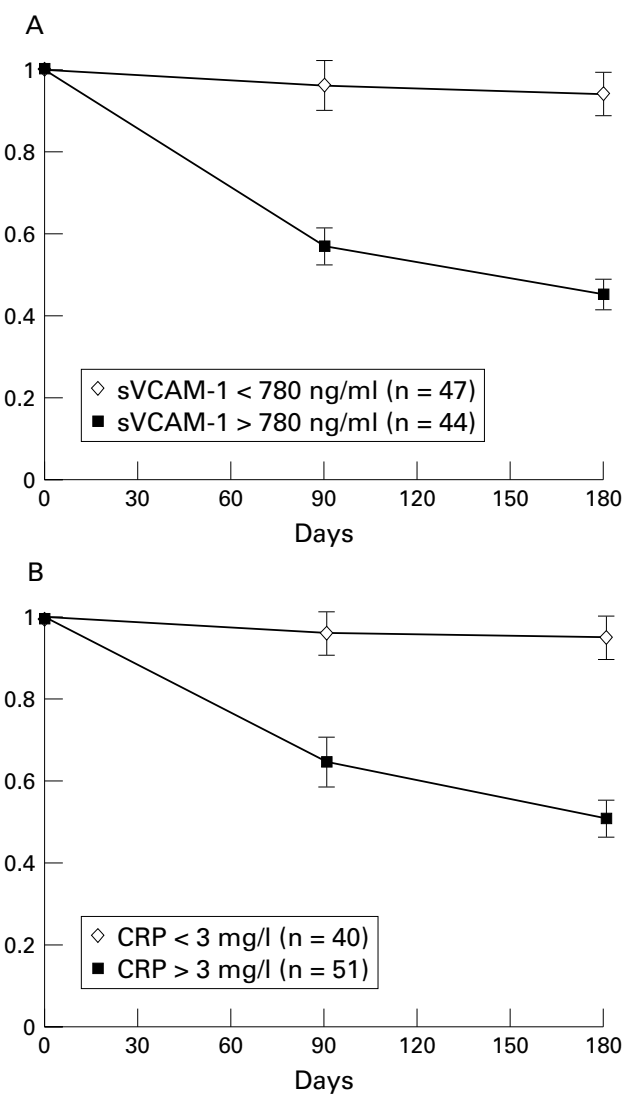

Figure 3 (A) Event free survival curves for patients divided according to concentration of $s$ VAM-1 $(<$ or $>$ $780 \mathrm{ng} / \mathrm{ml}$ ). (B) Event free survival curves for patients divided according to concentration of $C$ reactive protein (CRP) (<or $>3 \mathrm{mg} / \mathrm{l})$.

$>780 \mathrm{ng} / \mathrm{ml}$ and the ischaemic event rate for these patients was $55 \%$. The remaining 47 patients had sVCAM-1 concentrations $<780 \mathrm{ng} / \mathrm{ml}$, and the ischaemic event rate in this group was $6 \%$ (fig $3 \mathrm{~A}$ ). The sensitivity of a concentration of sVCAM-1 >780 ng/ml for predicting a future ischaemic event was $89 \%$ with a specificity of $69 \%$. The negative predictive value of sVCAM-1 $<780 \mathrm{ng} / \mathrm{ml}$ was $94 \%$.

\section{REACTIVE PROTEIN}

CRP concentrations were raised in patients who had an adverse event during follow up in comparison with those who did not (11.5 (4.2) v 5.4 (2.5) $\mathrm{mg} / \mathrm{l}, \mathrm{p}<0.001$, respectively) (fig 4). Concentrations of CRP remained significantly increased in the event group when we 


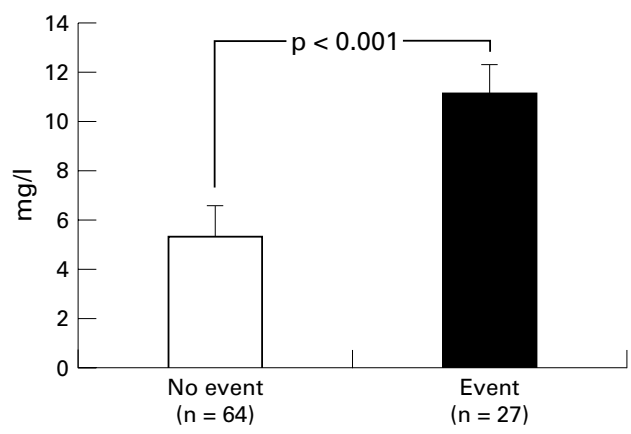

Figure 4 Concentrations of serum CRP at presentation in non-event and event groups.

added revascularisation as a combined end point compared with patients who had neither a follow up event nor revascularisation (8.7 (3.1) v 5.1 (3.6) mg/l, $\mathrm{p}<0.01$, respectively). Patients were divided according to CRP concentration $(<3 \mathrm{mg} / \mathrm{l} \text { or }>3 \mathrm{mg} / \mathrm{l})^{13}$ : CRP was $>3 \mathrm{mg} / 1$ in 51 patients $(56 \%)$ and the ischaemic event rate in these patients was $49 \%$ compared with an event rate of $5 \%$ in patients with a CRP concentration $<3 \mathrm{mg} / 1$ (fig 3B). The sensitivity of CRP $>3 \mathrm{mg} / 1$ for predicting a future ischaemic event was $96 \%$ with a specificity of $52 \%$. The negative predictive value of $\mathrm{CRP}<3 \mathrm{mg} / 1$ was $97 \%$. In a multiple regression analysis model both sVCAM-1 and CRP concentrations correlated with the occurrence of an ischaemic event over the duration of the study $(r=0.46, \mathrm{p}<0.01$ for $\mathrm{sVCAM}-1$ and $r=0.44, \mathrm{p}<0.01$ for CRP). There was no difference in the strength of correlation with ischaemic events between sVCAM-1 and CRP.

\section{Discussion}

The principal finding of this study was that concentrations of both SVCAM-1 and CRP are raised at the time of presentation in patients with UA and NQMI who go on to have major adverse cardiovascular events in the six months after initial presentation. Raised concentrations of both CRP and sVCAM-1 had a similar degree of sensitivity for predicting future ischaemic end points; however, the specificity of sVCAM-1 was higher than that of CRP (69\% $v 52 \%$, respectively).

SOLUBLE CELL ADHESION MOLECULES

Soluble CAMs are formed either by proteolytic cleavage of the extracellular portion of the molecule or, in the case of sP-selectin, by alternative splicing of the messenger RNA encoding for the entire transmembrane molecule. ${ }^{16}$ The exact pathophysiological role for these soluble CAMs has not been defined. In vitro studies have shown that concentrations of soluble CAMs in the supernatant of cultured endothelial cells directly reflect cell surface expression. ${ }^{17}$ Release of the extracellular portion from the cell surface may be a mechanism for down regulating cytokine induced increases in expression. These soluble molecules may function as competitive inhibitors of membrane bound forms, thereby regulating cell adhesion. This has been demonstrated for a soluble form of P-selectin, which is able to inhibit the adhesion of activated neutrophils to endothelial cells. ${ }^{18}$ However, to date, no definite biological role has been defined for SICAM-1, sVCAM-1, and sE-selectin, and these circulating forms may simply reflect active endovascular inflammation.

INFLAMMATORY MARKERS

The identification and use of CRP as a serum prognostic marker in patients with acute coronary syndromes has been reported previously. ${ }^{11}$ Initially, raised concentrations of CRP were found to have short term prognostic value unrelated to myocardial cell damage or myocardial ischaemia (normal cardiac troponin concentrations) in patients with UA. Evidence of long term prognostic value of raised concentrations of CRP was reported in patients without documented coronary artery disease. ${ }^{19}$ More recently, it was shown that CRP remains raised for up to three months after acute coronary syndromes in patients with adverse clinical outcomes. ${ }^{19} 20$ The acute phase rise in CRP concentrations is a non-specific phenomenon reflecting cytokine mediated hepatic production, triggered by most forms of inflammation, infection, and tissue injury. Raised concentrations of CRP reflect increased production of interleukin 6 and interleukin 1, two cytokines that can also stimulate the increased surface expression and subsequent release into the circulation of CAMs. ${ }^{21}$

The present study confirms the results of previous investigators by showing that raised CRP concentrations $(>3 \mathrm{mg} / \mathrm{l})$ are predictive of an adverse outcome in patients with acute coronary syndromes and we report, for the first time, that sVCAM-1 is a useful serum marker for predicting future ischaemic events in the six months after presentation with UA or NQMI. ${ }^{11}$ The raised concentrations of CRP and sVCAM-1 in patients who had a major adverse cardiovascular event during the six months of follow up was independent of the conventional cardiovascular risk factors of hypertension, hypercholesterolaemia, smoking, and diabetes mellitus in a multiple regression analysis model. A rise in sVCAM-1 did not add significantly to the predictive accuracy of a raised CRP concentration (>3 mg/l). The production of both of these inflammatory markers is critically dependent on raised concentrations of interleukin 1 and interleukin 6, and therefore elevation of CRP and sVCAM-1 may simply reflect enhanced production of these cytokines in patients with adverse clinical outcomes. It remains to be elucidated why the concentrations of only sVCAM-1 and not the other soluble CAMs measured are predictive of future events.

Recent data from the CAPTURE (chimeric 7E3 antiplatelet therapy in unstable angina refractory to standard treatment) trial compared the six month cardiac risk of raised cardiac troponin $\mathrm{T}$ and $\mathrm{CRP}$ concentrations. The investigators reported that raised cardiac troponin $\mathrm{T}$ was predictive of $\mathrm{MI}$ and mortality in the first 72 hours whereas raised CRP was not. The cardiac troponin $\mathrm{T}$ positive patients also 
displayed more recurrent ischaemia. However, for the six month follow up period CRP was predictive of death and MI. Interestingly, the incidence of coronary restenosis at six months was not related to cardiac troponin $\mathrm{T}$ concentrations but was significantly related to raised CRP. Raised troponins provide information relating to the risk of death and MI in the period immediately following an ischaemic event whereas raised CRP provides independent prognostic information regarding risk of events over the following six months. ${ }^{22}$ Therefore, it appears that troponin elevations relate to the thrombotic burden of the acutely ruptured plaque whereas CRP concentrations and similarly sVCAM-1 concentrations relate to the intensity of vascular inflammation and the subsequent risk of cardiovascular events in the medium term.

SOLUBLE CAMS AND CARDIOVASCULAR EVENTS Increased concentrations of SICAM-1 and sE-selectin have been reported in patients with ischaemic heart disease in comparison with controls. ${ }^{23}{ }^{24}$ Ridker and colleagues reported that a raised concentration of SICAM-1 is an independent risk factor for future $\mathrm{MI}$ in healthy men. ${ }^{25}$ More recent data have shown that patients with stable ischaemic heart disease who suffer MI or cardiovascular death have raised serum concentrations of SICAM-1 and sVCAM-1. ${ }^{26}$ These accumulating data indicate that soluble CAMs are useful in identifying patients at high risk of future cardiovascular events among both healthy men and patients with stable coronary artery disease. We previously reported that concentrations of sICAM-1, sVCAM-1, and sE-selectin are raised at the time of presentation in patients with UA and NQMI, ${ }^{27}$ and that the concentrations of all three soluble CAMs remain raised throughout the acute phase of presentation and then continue to be raised for up to six months after the acute ischaemic event. ${ }^{1428}$ The present study is the first to show that raised concentrations of soluble CAMs are predictive of an adverse outcome in UA and NQMI.

\section{CONCLUSIONS}

It was found in this study that a raised concentration of sVCAM-1 at the time of presentation with either UA or NQMI is predictive of future ischaemic events and has a prognostic accuracy comparable with that of raised concentrations of CRP. These findings strengthen the evidence that an inflammatory process is critical in the pathogenesis of acute coronary syndromes and suggests that the intensity of the inflammatory response can influence clinical outcome in acute coronary syndromes. These observations have implications for clinical practice because inflammatory markers may facilitate risk stratification of patients with acute coronary syndromes during the acute phase of presentation.

This project has been generously supported by a grant from the Royal City of Dublin Hospital Board. We are grateful to Professor Dermot Kelleher and the staff of the Sir Patrick Dun's sor Dermot Kelleher and the staff of the Sir Patrick Dun's
Research Laboratory for their assistance and advice in this work.
1 Ross R. The pathogenesis of atherosclerosis: a perspective for the 1990's. Nature 1990;326:801-9.

2 Davies M, Thomas A. Plaque fissuring: the cause of acute myocardial infarction, sudden ischemic death, and crescendo angina. Br Heart f 1985;53:363-73.

3 Fuster V, Badimon L, Badimon J, et al. The pathogenisis of coronary artery disease and the acute coronary syndromes. N Engl f Med 1992;326:242-50.

4 Ritchie ME. Nuclear factor-kB is selectively and markedly activated in humans with unstable angina pectoris. Circulation 1998;98:1707-13.

5 Van der Wal A, Becker A, van der Loos C, et al. Site of intimal rupture or erosion of thrombosed coronary atherosclerotic plaques is characterised by an inflammatory process irrespective of the dominant plaque morphology. Circulation 1994;89:36-44.

6 Mazzone A, De Servi S, Ricevuti G. Increased expression of neutrophil and monocyte adhesion molecules in unstable coronary artery disease. Circulation 1993;88:358-63.

7 Dinerman JL, Metha JL, Saldeen TG, et al. Increased neutrophil elastase release in unstable angina pectoris and acute myocardial infarction. F Am Coll Cardiol 1990;15: 1559-63.

8 Hirsh PD, Hillis LD, Campbell WB, et al. Release of prostaglandins and thromboxane into the coronary circulation in patients with ischaemic heart disease. $N$ Engl f Med 1981;304:685-91.

9 Berk BC, Weintraub WS, Alexander RW. Elevation of C-reactive protein in "active" coronary artery disease. $A m \mathcal{F}$ Cardiol 1990;65:168-72.

10 de Beer FC, Hind CRK, Fox KM, et al. Measurement of serum C-reactive protein concentration in myocardial ischaemia and infarction. Br Heart f 1982;47:239-43.

11 Liuzzo G, Biasucci LM, Gallimore JR, et al. The prognostic value of C-reactive protein and serum amyloid A protein in severe unstable angina. N Engl F Med 1994;331:417-24.

12 Jang Y, Lincoff A, Plow E, et al. Cell adhesion molecules in coronary artery disease. F Am Coll Cardiol 1994;24:1591601.

13 Gearing AJH. Circulating adhesion molecules in disease. Immunol Today 1993;14:506.

14 Mulvihill N, Foley JB, Ghaisas N, et al. Soluble adhesion molecule expression in acute ischaemic syndromes [abstract]. F Am Coll Cardiol 1999;33:385A

15 Braunwald E. Unstable angina: a classification. Circulation 1989;80:410-4.

16 Ishiwata N, Takio K, Katayama M, et al. Alternatively spliced isoform of P-selectin is present in vivo as a soluble molecule. F Biol Chem 1994;269:23708-15.

17 Piggot R, Dillon LP, Hemingway IH, et al. Soluble forms of E-selectin, ICAM-1 and VCAM-1 in the supernatants of cytokine activated cultured endothelial cells. Biochem Biophys Res Commun 1992;187:584-9.

18 Gamble JR, Skinner MP, Berndt MC, et al. Prevention of activated neutrophil adhesion to endothelium by soluble adhesion protein GMP-140. Science 1990;249:414-7.

19 Biasucci LM, Luizzo G, Grillo RL, et al. Elevated levels of $\mathrm{C}$-reactive protein at discharge in patients with unstable angina predict recurrent instability. Circulation 1999;99: $855-60$

20 Caligiuri G, Luizzo G, Biasucci LM, et al. Immune system activation follows inflammation in unstable angina: pathogenetic implications. F Am Coll Cardiol 1998;32: 1295-304.

21 Munro M. Endothelial-leukocyte adhesive interactions in inflammatory diseases. Eur Heart f 1993;14(suppl):72-7.

22 Heeschen C, Hamm CW, Bruemmer J, et al. Predictive value of CRP and troponin-T in patients with unstable angina: a comparative analysis. CAPTURE Investigators. $\mathcal{F}$ Am Coll Cardiol 2000;35:1535-42.

23 Haught WH, Mansour M, Rothlein R, et al. Alterations in circulating intercellular adhesion molecule-1 and L-selectin: further evidence for chronic inflammation in ischemic heart disease. Am Heart f 1996;132:1-8.

24 Blann AD, Amiral J, McCollum C. Circulating endothelial cell/leucocyte adhesion molecules in ischaemic heart disease. Br F Haematol 1996;95:263-5.

25 Ridker P, Hennekens CH, Roitman-Johnson B, et al. Plasma concentrations of soluble intercellular adhesion molecule 1 and risks of future myocardial infarction in apparently healthy men. Lancet 1998;351:88-92.

26 Wallen NH, Held C, Rehnqvist N, et al. Elevated serum intercellular adhesion molecule-1 and vascular cell adhesion molecule-1 among patients with stable angina pectoris who suffer cardiovascular death or non-fatal myocardial infarction. Eur Heart F 1999;20:1039-43.

27 Mulvihill N, Foley JB, Ghaisas N, et al. Early temporal expression of soluble cellular adhesion molecules in patients with unstable angina and subendocardial myocardial infarction. Am f Cardiol 1999;83:1265-7.

28 Mulvihill N, Foley B, Ghaisas N, et al. Evidence of prolonged inflammation in unstable angina and non Q-wave myocardial infarction. $\mathcal{F}$ Am Coll Cardiol 2000;34: 1210-6. 